\title{
Clinical applicability of the Feline Grimace Scale: real-time versus image scoring and the influence of sedation and surgery
}

\author{
Marina C Evangelista ${ }^{1}$, Javier Benito ${ }^{1}$, Beatriz P Monteiro ${ }^{1}$, Ryota Watanabe ${ }^{1}$, Graeme M Doodnaught ${ }^{1}$, Daniel \\ Pang $^{1,2}$, Paulo V Steagall ${ }^{\text {Corresp. } 1}$ \\ 1 Department of Clinical Sciences, Faculty of Veterinary Medicine, Université de Montréal, St-Hyacinthe, Quebec, Canada \\ ${ }^{2}$ Veterinary Clinical and Diagnostic Sciences, Faculty of Veterinary Medicine, University of Calgary, Calgary, Alberta, Canada \\ Corresponding Author: Paulo V Steagall \\ Email address: paulo.steagall@umontreal.ca
}

Background. The Feline Grimace Scale (FGS) is a facial expression-based scoring system for acute pain assessment in cats with reported validity using image assessment. The aims of this study were to investigate the clinical applicability of the FGS in real-time when compared with image assessment, and to evaluate the influence of sedation and surgery on FGS scores in cats. Methods. Sixty-five female cats (age: $1.37 \pm 0.9$ years and body weight: $2.85 \pm 0.76 \mathrm{~kg}$ ) were included in a prospective, randomized, clinical trial. Cats were sedated with intramuscular acepromazine and buprenorphine. Following induction with propofol, anesthesia was maintained with isoflurane and cats underwent ovariohysterectomy $(\mathrm{OVH})$. Pain was evaluated at baseline, 15 minutes after sedation, and at $0.5,1,2,3,4,6,8,12$ and 24 hours after extubation using the FGS in real-time (FGSRT). Cats were video-recorded simultaneously at baseline, 15 minutes after sedation, and at $2,6,12$, and 24 hours after extubation for subsequent image assessment (FGS-IMG), which was performed six months later by the same observer. The agreement between FGS-RT and FGS-IMG scores was calculated using the Bland \& Altman method for repeated measures. The effects of sedation (baseline versus 15 minutes) and OVH (baseline versus 24 hours) were assessed using linear mixed models. Responsiveness to the administration of rescue analgesia (FGS scores before versus one hour after) was assessed using paired ttests. Results. Minimal bias (-0.057) and narrow limits of agreement (-0.351 to 0.237$)$ were observed between the FGS-IMG and FGS-RT. Scores at baseline (FGS-RT: $0.16 \pm 0.13$ and FGS-IMG: $0.14 \pm 0.13$ ) were not different after sedation (FGS-RT: $0.2 \pm 0.15, p=0.39$ and FGS-IMG: $0.16 \pm 0.15, p=0.99$ ) nor at 24 hours after extubation (FGS-RT: $0.16 \pm$ $0.12, p=0.99$ and FGS-IMG: $0.12 \pm 0.12, p=0.96$ ). Thirteen cats required rescue analgesia; their FGS scores were lower one hour after analgesic administration (FGS-RT: $0.21 \pm 0.18$ and FGS-IMG: $0.18 \pm 0.17$ ) than before (FGS-RT: $0.47 \pm 0.24, p=0.0005$ and 
FGS-IMG: $0.45 \pm 0.19, p=0.015)$. Conclusions. Real-time assessment slightly overestimates image scoring; however, with minimal clinical impact. Sedation with acepromazine-buprenorphine and ovariohysterectomy using a balanced anesthetic protocol did not influence the FGS scores. Responsiveness to analgesic administration was observed with both the FGS-RT and FGS-IMG. 
1 Clinical applicability of the Feline Grimace Scale: real-

2 time versus image scoring and the influence of

3 sedation and surgery

4

5

6

7

8

Marina C. Evangelista ${ }^{1}$, Javier Benito ${ }^{1}$, Beatriz P. Monteiro ${ }^{1}$, Ryota Watanabe ${ }^{1}$, Graeme M. Doodnaught $^{1}$, Daniel S. J. Pang ${ }^{1,2}$, Paulo V. Steagall ${ }^{1}$

${ }^{1}$ Department of Clinical Sciences, Faculty of Veterinary Medicine, Université de Montréal, Saint-Hyacinthe, QC, Canada

${ }^{2}$ Veterinary Clinical and Diagnostic Sciences, Faculty of Veterinary Medicine, University of Calgary, Calgary, Alberta, Canada

Corresponding Author:

Paulo V Steagall ${ }^{1}$

3200 rue Sicotte, Saint-Hyacinthe, QC, J2S 2M2, Canada

Email address: paulo.steagall@umontreal.ca

\section{Abstract}

Background. The Feline Grimace Scale (FGS) is a facial expression-based scoring system for acute pain assessment in cats with reported validity using image assessment. The aims of this study were to investigate the clinical applicability of the FGS in real-time when compared with image assessment, and to evaluate the influence of sedation and surgery on FGS scores in cats. Methods. Sixty-five female cats (age: $1.37 \pm 0.9$ years and body weight: $2.85 \pm 0.76 \mathrm{~kg}$ ) were included in a prospective, randomized, clinical trial. Cats were sedated with intramuscular acepromazine and buprenorphine. Following induction with propofol, anesthesia was maintained with isoflurane and cats underwent ovariohysterectomy $(\mathrm{OVH})$. Pain was evaluated at baseline, 15 minutes after sedation, and at $0.5,1,2,3,4,6,8,12$ and 24 hours after extubation using the FGS in real-time (FGS-RT). Cats were video-recorded simultaneously at baseline, 15 minutes after sedation, and at 2, 6, 12, and 24 hours after extubation for subsequent image assessment (FGS-IMG), which was performed six months later by the same observer. The agreement between FGS-RT and FGS-IMG scores was calculated using the Bland \& Altman method for repeated measures. The effects of sedation (baseline versus 15 minutes) and $\mathrm{OVH}$ (baseline versus 24 hours) were assessed using linear mixed models. Responsiveness to the administration of rescue analgesia (FGS scores before versus one hour after) was assessed using paired t-tests. Results. Minimal bias ( -0.057$)$ and narrow limits of agreement ( -0.351 to 0.237$)$ were observed between the FGS-IMG and FGS-RT. Scores at baseline (FGS-RT: $0.16 \pm 0.13$ and FGS-IMG: $0.14 \pm 0.13$ ) were not different after sedation (FGS-RT: $0.2 \pm 0.15, p=0.39$ and FGS-IMG: 0.16 $\pm 0.15, p=0.99$ ) nor at 24 hours after extubation (FGS-RT: $0.16 \pm 0.12, p=0.99$ and FGS-IMG: 
$400.12 \pm 0.12, p=0.96)$. Thirteen cats required rescue analgesia; their FGS scores were lower one 41 hour after analgesic administration (FGS-RT: $0.21 \pm 0.18$ and FGS-IMG: $0.18 \pm 0.17$ ) than

42 before (FGS-RT: $0.47 \pm 0.24, p=0.0005$ and FGS-IMG: $0.45 \pm 0.19, p=0.015$ ).

43 Conclusions. Real-time assessment slightly overestimates image scoring; however, with

44 minimal clinical impact. Sedation with acepromazine-buprenorphine and ovariohysterectomy

45 using a balanced anesthetic protocol did not influence the FGS scores. Responsiveness to

46 analgesic administration was observed with both the FGS-RT and FGS-IMG.

47

48

49

50

51

52

53

54

55

56

57

58

59

60

61

62

63

64

65

66

67

68

69

70

71

Introduction

9

(5)

and under treated in cats worldwide (Lorena et al. 2014; Hunt et al. 2015; Reimann et al. 2017; Morales-Vallecilla et al. 2019). Facial expressions are considered a reliable method of pain assessment in non-verbal humans and other mammals (Prkachin, 2009; Langford et al. 2010; Sotocinal et al. 2011; Dalla Costa et al. 2014; Finka et al. 2019).

5

56
Linear distances between specific facial landmarks and the quantification of changes in
facial shape for the study of pain in cats have been published (Holden et al. 2014; Finka et al. 2019). Recently, a facial expression-based scoring system has been proposed for assessing acute pain in cats, namely the Feline Grimace Scale (FGS). This instrument has been developed and validated in cats with different sources and intensities of pain produced by naturally-occurring conditions; additionally, its reliability, criterion and construct validity (including responsiveness) were assessed using image scoring (Evangelista et al. 2019).

2
The standard methodology for assessing facial expressions of pain using grimace scales
usually relies on image scoring (Langford et al. 2010; Sotocinal et al. 2011; Dalla Costa et al. 2014). The images are commonly screenshots obtained from videos which represents a timeconsuming procedure that takes place weeks or months after video-recordings. In clinical practice, pain must be promptly assessed to ensure appropriate and immediate treatment. Realtime scoring has been described using the Mouse Grimace Scale (MGS; Miller \& Leach, 2015) and the Rat Grimace Scale (RGS; Leung et al. 2016), but not the FGS.

Elective sterilization of female companion animals (i.e. ovariohysterectomy, OVH) is one of the most common surgical procedures performed in veterinary medicine. The administration of sedatives, anesthetics and analgesics are required in the perioperative setting and drugs such as ketamine have been shown to produce confounding effects by increasing psychomotor scores with feline pain scales (Buisman et al. 2016). Indeed, the influence of anesthesia, surgery and opioids (i.e. buprenorphine) on grimace scale scores have been demonstrated in mice and rats (Miller et al. 2015; Leung et al. 2016). Changes in facial shape have been reported in cats after OVH (Finka et al. 2019). It is not known how sedation and OVH itself may affect FGS scores, 78 clinical practice. 
79

80

81

82

83

84

85

86

87

88

89

90

91

92

93

94

95

96

97

98

99

100

101

102

103

104

105

106

107

108

109

110

111

112

113

114

115

116

117

118

The objectives of this study were: 1) to investigate whether the FGS could be successfully implemented in a clinical setting in cats undergoing $\mathrm{OVH}$, enabling real-time pain assessment; 2) to evaluate the influence of sedation and OVH on the FGS; and 3) to reassess the responsiveness to analgesic treatment of the FGS in real-time. Our hypotheses were: 1) FGS scores obtained in real-time and by image assessment would be comparable when evaluated by the same observer; 2) FGS scores would not change after sedation with acepromazine and buprenorphine or at 24 hours after extubation when compared with baseline values; and 3) the FGS scores in real-time would decrease after analgesic treatment.

\section{Materials \& Methods}

\section{Animals}

Eighty-one adult female domestic cats from local shelters were admitted to the veterinary teaching hospital (Centre Hospitalier Universitaire Vétérinaire) of the Faculty of Veterinary Medicine, Université de Montréal for elective OVH between June and October 2018. The study was designed to evaluate the analgesic efficacy of intraperitoneal administration of bupivacaine alone or in combination with dexmedetomidine (Benito et al. 2019). The current study occurred in parallel to evaluate changes in the FGS. The protocol was approved by the local animal care committee, Comité d'éthique de l'utilisation des animaux - Université de Montréal (protocol number 18-Rech-1825). Written informed consent for participation in the study was obtained for each patient.

Inclusion criteria included healthy cats based on physical examination and hematology. Exclusion criteria included cats presenting with cardiac arrhythmias, body condition score $>7$ or $<3$ on a scale from 1 to 9 , anemia (hematocrit $<25 \%$ ), hypoproteinemia (total protein $<5.9$ $\mathrm{g} / \mathrm{dL}$ ), cats with high pain scores at presentation (see pain assessment), or any clinical signs of diseases during physical examination such as upper respiratory tract disease and conjunctivitis. Cats were admitted the day before the procedures and discharged 24 hours after the surgery. They were housed individually in adjacent cages in a cat-only ward with free access to water and food, and a litter box. Environmental enrichment included hanging toys, blankets and a cardboard box that cats could use as a hiding spot or as an elevated surface.

\section{Anesthesia and surgery}

Anesthetic and surgical procedures are described thoroughly elsewhere (Benito et al. 2019). In brief, food was withheld for $8-12$ hours before general anesthesia; cats were premedicated with acepromazine $(0.02 \mathrm{mg} / \mathrm{kg})$ and buprenorphine $(0.02 \mathrm{mg} / \mathrm{kg})$ intramuscularly; anesthesia was induced with propofol intravenously to allow endotracheal intubation $(4-6 \mathrm{mg} / \mathrm{kg})$ and maintained with isoflurane in oxygen. Cats were randomly assigned to receive one of the two following treatments: bupivacaine $(2 \mathrm{mg} / \mathrm{kg}$ ) or bupivacaine (same dose) with 
119 dexmedetomidine $(1 \mu \mathrm{g} / \mathrm{kg})$. All cats received an intraperitoneal administration of either

120 bupivacaine alone or in combination with dexmedetomidine intraoperatively. The intraperitoneal

121

122

123

124

125

126

127

128

129

130

131

132

133

134

135

136

137

138

139

140

141

142

143

144

145

146

147

148

149

150

151

152

153

154

155

156

157

158 sterile solution was instilled (splashed) before OVH by the veterinary surgeon, over the right and left ovarian pedicles, and the caudal aspect of the uterine body in three equal volumes. Ovariohysterectomy was performed approximately one minute after intraperitoneal administration using the pedicle tie technique. All cats were discharged the day after surgery and returned to their respective shelters for adoption.

\section{Sedation and pain assessment}

Sedation was assessed using a 5-point simple descriptive scale, where $0=$ no sedation, 1 $=$ able to stand but is wobbly; $2=$ in sternal recumbency; $3=$ can lift its head; $4=$ fast asleep $/$ no response to hand clap (adapted from Steagall et al. 2009).

Pain was evaluated using the short-form UNESP-Botucatu composite pain scale (SFUBCPS) and the FGS. The SF-UBCPS is a novel pain scoring system consisting of 4 items (each item is scored from 0 to 3 adding up to a maximum score of 12 points) to evaluate the cats' posture, activity, attitude and reaction to touch and palpation of a painful site. This scale has been validated using video-based assessments (Steagall P, 2019, unpublished data). Cats with a baseline pain score of $\geq 2$ using the SF-UBCPS were not included in the study. High baseline scores could indicate a mild degree of pain before the surgery, or an influence of the cat's demeanor (shy, fearful or feral) (Buisman et al. 2017). The FGS consists of five action units (ear position, orbital tightening, muzzle tension, whiskers change and head position), each one is scored from 0 to $2(0=$ action unit is absent; $1=$ moderate appearance of the action unit, or uncertainty over its presence; and $2=$ obvious appearance of the action unit) (Fig. 1). If one or more action units were not visible, the observer had the option of marking "not possible to score" and the weight was redistributed to the other action units. A total FGS score was calculated by the sum of the scores of the action units divided by the total possible score, excluding those marked as not possible to score (e.g. $4 / 8=0.5$ ). Images with more than 2 "not possible to score" were excluded from final analysis.

Sedation and pain assessments were performed by one observer (MCE) at baseline (morning before the surgery), 15 minutes after premedication, and at $0.5,1,2,3,4,6,8,12$ and 24 hours after extubation. Sedation was evaluated before pain assessment. The FGS scores were obtained in real time (FGS-RT) using instantaneous sampling during three minutes of observation without interacting with the cat. A stopwatch was set to control the time, and at the end of each minute (first, second and third minutes), one score per action unit was assigned (representing the whole minute of observation) and a total FGS-RT score was obtained. The average of those three scores was considered the FGS-RT score for that time-point [i.e. $(0.5+0.4$ $+0.5) / 3=0.47]$. Three-minute videos were recorded simultaneously during the FGS-RT assessment using a high-definition video camera (GoPro Hero 5, GoPro Inc., San Mateo, CA, USA) at the following time-points: baseline, 15 minutes after premedication, and at 2, 6, 12 and 
159

160

161

162

163

164

165

166

167

168

169

170

171

172

173

174

175

176

177

178

179

180

181

182

183

184

185

186

187

188

189

190

191

192

193

194

195

196

197

198

24 hours after extubation (Fig. 2). The camera was placed between the cage bars at the level of the cats' eyes and set to record at 60 frames per second and a medium angle of view. Following the three-minute recording and FGS-RT scoring, the observer proceeded with the interaction and palpation of the wound/painful area to complete the SF-UBCPS.

Rescue analgesia was provided with buprenorphine $0.02 \mathrm{mg} / \mathrm{kg}$ intravenously and meloxicam $0.2 \mathrm{mg} / \mathrm{kg}$ subcutaneously when SF-UBCPS $\geq 4$ (the analgesic intervention threshold pre-determined for this scale). Additional rescue analgesia was administered if needed, with the same dose of buprenorphine. If a cat required the administration of rescue analgesia, additional videos (along with the pain evaluation using the FGS-RT and SF-UBCPS) were recorded before and one hour after its administration. If a cat did not require the administration of rescue analgesia before the $12 \mathrm{~h}$ time-point, meloxicam was administered (same dose and route of administration as previously described for rescue analgesia). The choice of administering meloxicam at $12 \mathrm{~h}$ for the cats that did not require rescue analgesia beforehand is explained by the time gap until the $24 \mathrm{~h}$ evaluation and we wanted to make sure cats were not painful overnight.

\section{Video handling, image selection and image scoring}

The videos were downloaded into a computer and renamed after a random sequence of numbers (www.random.org). Videos were not recorded postoperatively if the cats had been previously spayed. In these cases, only videos recorded at baseline and 15 minutes after sedation were considered. Any identification of the cats' names or time-points were deleted for blinding purposes. Screenshots were obtained from video-recordings whenever the cat was facing the camera, but not when they were sleeping, grooming or vocalizing. The resulting images were identified as belonging to the first, second or third minute of the video. From each three-minute video, the single best image per minute was selected for later scoring. Images were not selected if quality was poor (i.e. blurred or dark images or when the cats' faces were partially/completely not visible).

The same observer (MCE) evaluated pain in real-time using the FGS followed by the SFUBCPS, and scored the images using the FGS (FGS-IMG) in a blinded and randomized order, six months after the experimental study. The average score of the three images (each from the first, second and third minutes) was considered the final FGS-IMG score for that time-point.

\section{Statistical analysis}

Statistical analyses were performed with SAS v.9.3 (SAS Institute, Cary, NC, USA) and GraphPad Prism 7 (GraphPad software, San Diego, CA, USA).

The agreement between FGS-RT and FGS-IMG scores was assessed per minute of observation and for the average of three minutes using the Bland \& Altman's method for repeated measures (Bland \& Altman, 2007). This method describes the agreement between two 
199

200

201

202

203

204

205

206

207

208

209

210

211

212

213

214

215

216

217

218

219

220

221

222

223

224

225

226

227

228

229

230

231

232

233

234

235

236

237

238

quantitative measurements by evaluating a bias between the mean differences, and estimating an agreement interval, within which $95 \%$ of the differences fall.

Sedation scores (ordinal variable) over time were compared with baseline using a nonparametric approach (Friedman test and Dunn's multiple comparisons test). Sedation scores are presented as median (range).

Linear mixed models with individuals as random effect and time as fixed effect were used to evaluate the time course of the FGS-RT, and the influence of sedation (baseline versus 15 min after premedication) and OVH (baseline versus $24 \mathrm{~h}$ after extubation) on both total FGSRT and FGS-IMG scores (considered continuous variables). Scores obtained after the administration of rescue analgesia were not included in this analysis. FGS-IMG and FGS-RT scores are presented as mean \pm standard deviation (SD). Only the FGS-RT scores were used for the time course evaluation because scores from all cats and all time-points were available. FGSIMG scores were obtained at fewer time-points and they were sometimes missing due to the lack of acceptable images from some videos.

The Cochran-Mantel-Haenszel test for ordinal scores and repeated measures was used to assess the influence of sedation and $\mathrm{OVH}$ on each individual action unit (scores 0,1 or 2). This approach takes the order into account, thus representing a more specific test and offering greater statistical power.

Responsiveness to rescue analgesia (i.e. construct validity) was assessed by comparing the scores before and one hour after the administration of buprenorphine and meloxicam. In this case, after confirming the normality of data distribution using the Shapiro Wilk test, FGS-RT and FGS-IMG scores were compared using paired t-tests. The SF-UBCPS scores (ordinal variable and not normally distributed) were compared using the Wilcoxon test. SF-UBCPS scores are presented as median (range).

$P$ values were adjusted according to the number of comparisons for each analysis. Bonferroni-corrected values of $p<0.05$ were considered significant.

\section{Results}

Sixty-five mixed-breed cats met the inclusion criteria (age: $1.37 \pm 0.9$ years and body weight: $2.85 \pm 0.76 \mathrm{~kg}$; domestic short-haired: $\mathrm{n}=52$ and domestic long-haired cats: $\mathrm{n}=13$ ). Sixteen cats were excluded because of upper respiratory tract disease $(n=4)$, conjunctivitis $(n=$ 1), facial hemiparalysis $(n=1)$, anemia and hypoproteinemia $(n=1)$, body condition score $>7 / 9$ $(n=1)$ and $<3 / 9(n=1)$ or high baseline SF-UBCPS scores $(n=7)$.

A total of 394 videos and 1373 still images were recorded and obtained, respectively. The median (range) number of selected images per cat was 6 (0-20). The maximum number of possible still images selected per cat would be 18 if this cat did not require rescue analgesia, considering that it could be possible to select one image from each minute for each video of 3 minutes recorded at the six planned time-points (baseline, $15 \mathrm{~min}, 2 \mathrm{~h}, 6 \mathrm{~h}, 12 \mathrm{~h}$ and 24$)(3 \times 6=$ 18). Following the same reasoning, if the cat required rescue (i.e. at $4 \mathrm{~h}$ ), two additional videos 
239 would be recorded (at $4 \mathrm{~h}$ and $5 \mathrm{~h}$ ) and the maximum number of possible images would be 20 (18 $240+2=20$ ).

241 The total number of images used for scoring was 540. It was not possible to obtain any 242 image from 160 videos. The selected images $(n=540)$ were scored in four sessions of three 243 hours maximum (135 images per day, every other day) to avoid observer fatigue.

244

245

246

247

248

249

250

251

252

253

254

255

256

257

258

259

260

261

262

263

264

265

266

267

268

269

270

271

272

273

274

275

276

277

278

\title{
Agreement FGS-RT and FGS-IMG
}

Minimal bias and narrow limits of agreement (LOA) were observed between the FGS-RT and FGS-IMG scores. The agreement was calculated for each minute of observation (Fig. 3A3C) and for the average of three minutes (final score per time-point; Fig. 3D). The FGS-RT scores overestimated FGS-IMG scores (Bias ${ }_{1 \text { st } \min }=-0.078$, Bias ${ }_{2 \text { nd } \min }=-0.054$, Bias 3 rd $\min =-$ 0.050 and Bias final score $=-0.057$ ).

\section{Sedation scores}

\begin{abstract}
Sedation scores were significantly higher than baseline at $15 \mathrm{~min}$ after sedation $(p=$ $0.012)$ and $0.5 \mathrm{~h}$ after extubation $(p<0.001)$ (Table 1$)$.
\end{abstract}

\section{Time course and influence of sedation and OVH on FGS scores}

In comparison with baseline, mean \pm SD FGS-RT scores were not different at 15 minutes after sedation, nor at $0.5 \mathrm{~h}$ and $24 \mathrm{~h}$ after extubation. The FGS-RT scores were significantly higher than baseline from 1 to $12 \mathrm{~h}$ after extubation (Table 2 and Fig. 4).

In comparison with baseline, mean \pm SD FGS-IMG scores $(0.14 \pm 0.13)$ were not different at 15 minutes after sedation $(0.16 \pm 0.15$, mean difference $=0.028,95 \% \mathrm{CI}$ of the differences: -0.0298 to $0.0864, p=0.9911)$ nor at $24 \mathrm{~h}$ after extubation $(0.12 \pm 0.12$, mean difference $=-0.033,95 \% \mathrm{CI}$ of the differences: -0.1 to $0.0339, p=0.9569$ ).

\section{Influence of sedation and OVH on individual action units}

Sedation did not influence final FGS scores (both -RT and -IMG); however, orbital tightening scores obtained in real-time 15 minutes after sedation were higher than baseline ( $p=$ 0.026 ). This effect was not observed with any other action unit (ear position: $p=0.72$; muzzle tension: $p=0.17$; whiskers change: $p=0.58$; head position: $\mathrm{p}=0.22$ ). Similarly, no significant differences in FGS-IMG scores were observed after sedation (ear position: $p=0.1$; orbital tightening: $p=0.32$; muzzle tension: $p=0.14$; whiskers change: $p=0.17$; head position: $p=$ $0.61)$.

Ovariohysterectomy using a balanced anesthetic protocol did not influence any action unit scores obtained using either FGS-RT or FGS-IMG. All action units scores $24 \mathrm{~h}$ after 
279

280

281

282

283

284

285

286

287

288

289

290

291

292

293

294

295

296

297

298

299

300

301

302

303

304

305

306

307

308

309

310

311

312

313

314

315

316

317

318

extubation were not significantly different from baseline values (FGS-RT - ear position: $p=$ 0.076; orbital tightening: $p=0.35$; muzzle tension: $p=0.72$; whiskers change: $p=0.62$; head position: $p=0.35$ and FGS-IMG - ear position: $p=0.16$; orbital tightening: $p=0.22$; muzzle tension: $p=0.12$; whiskers change: $p=0.1$; head position: $p=0.93$ ).

\section{Rescue analgesia}

Thirteen cats required rescue analgesia throughout the postoperative period. Mean \pm SD scores before and after rescue analgesia were $0.47 \pm 0.24$ and $0.21 \pm 0.18$ (mean difference $=$ $0.266,95 \% \mathrm{CI}$ of the differences: -0.389 to $-0.143, p=0.0005)$ respectively, using FGS-RT assessment; and $0.45 \pm 0.19$ and $0.18 \pm 0.17$ (mean difference $=-0.274,95 \% \mathrm{CI}$ of the differences: -0.481 to $-0.0681, p=0.0154$ ) respectively, using FGS-IMG assessment. Median (range) SF-UBCPS scores before and after analgesia were 7 (5-9) and 1 (0-3), respectively (median of differences: $-3,95 \% \mathrm{CI}$ of the differences: -4 to $-3, p=0.0005$ ).

\section{Discussion}

This study demonstrated the applicability of the Feline Grimace Scale in a clinical setting using real-time scoring. Minimal bias and narrow LOA were observed between scoring methods; the sedation protocol and surgery did not influence FGS scores and both FGS-RT and FGS-IMG detected responsiveness to analgesic administration.

The Bland \& Altman for repeated measures method was used to verify the applicability of the FGS in real-time in a clinical setting. Real-time scoring using the FGS slightly overestimated image assessment (Bias final score: -0.057). This could be explained by the clinical context of FGS-RT scoring and the three-minute observation of the cats' facial expression. Although the observer was focused on the action units to be scored, the body position and posture of the cat could be observed, and this might be another reason for the overestimation with the FGS-RT scores. Additionally, the FGS-IMG scores reflected the pain states using only one still image selected during that minute. It is possible that facial expressions might not be the exact same if an image was selected at the beginning or the end of each minute of observation, which may also explain the discrepancy observed with the FGS-RT when compared with FGSIMG. Real-time scoring using the RGS was also demonstrated feasible with good agreement between real-time and image scoring (Leung et al. 2016). In contrast to what has been observed in rats, real-time MGS scores were significantly lower than those obtained by image assessment in mice (Miller \& Leach, 2015). In both rodent species, real-time scoring underestimated the scores obtained through image assessment. These differences might be related to the procedure for image capture. In rats, similar methodology was used; however, multiple intervals and punctual observations for RGS scoring methods were tested in real-time (Leung et al. 2016). In mice, photographs were taken to obtain the MGS scores (Miller \& Leach, 2015). In the latter study, the authors speculated that the use of photographs may have resulted in an artificial 
319 elevation of scores by capturing specific behaviors or movements (such as blinking), and that

320

321

322

323

324

325

326

327

328

329

330

331

332

333

334

335

336

337

338

339

340

341

342

343

344

345

346

347

348

349

350

351

352

353

354

355

356

357

358

some difficulty in real-time scoring could be expected by their constant (and fast) level of activity. For the FGS, screenshots were taken from the videos when the cat was facing the camera. It is possible that the screenshots were obtained when the cat was paying attention to the surroundings (eyes open and ears facing forward) thus decreasing the resulting FGS-IMG score. Additionally, during FGS-RT scoring, the observer was aware of the timing (pre- or postoperatively). Although the non-blind nature of real-time assessment is inevitable and could represent a source of bias, it represents the method by which pain is evaluated in the clinical setting. Moreover, the good agreement between IMG and RT scores implies that the fact of the observer being aware of the timing did not introduce substantial bias.

There is no consensus for the classification of the LOA for pain scores with the same degree of standardization that exists for physiological variables (e.g. blood pressure, cardiac output, blood gas measurements, etc.) when designing measurement comparison studies (Mantha et al. 2000). As in the case of blood pressure monitors, the American National Standards of the Association for the Advancement of Medical Instrumentation recommends a limit for the mean difference of the paired measurements (bias) of $\pm 5 \mathrm{mmHg}$ or less between the test system and the comparison system (White et al. 1993). These criteria are not readily applicable for pain scores. Alternatively, the evaluation of the LOA in relation to the analgesic threshold has been proposed (Leung et al. 2016). The LOA observed in the present study, although narrow (LOA final score: -0.351 to 0.237 ), spans the analgesic threshold of 0.39 out of 1.0 previously determined for the FGS (Evangelista et al. 2019). This would mean that FGS-RT scores that are close to the analgesic threshold (slightly lower or higher than 0.39 ) should be interpreted with caution since this threshold is a suggestion for the administration of rescue analgesia based on the probability of being painful above that score. However, this should not be a major clinical problem if the final decision for giving additional analgesics rely on clinical judgement while taking in consideration the FGS score, context and disease, and the potential reassessment of the cat after a brief period.

Considering the small average discrepancy between methods for bias (with consistent variability for most of the FGS score range) and narrow LOA, the methods (FGS-IMG and FGSRT) would probably provide similar assessments in the clinical setting. It should be noted; however, that the authors did not define the LOA a priori to state that real time and image assessments are interchangeable.

Although the assessment can be difficult in patients under the effects of anesthetics and sedatives, pain must be evaluated in a valid and reliable manner to ensure adequate treatment. For example, it is known that ketamine-based protocols confound pain scores in cats using the UNESP Botucatu Multidimensional Composite Pain Scale (Buisman et al. 2016). In this study, the premedication did not seem to affect FGS scores. Sedation scores increased after premedication with acepromazine-buprenorphine; however, it did not influence FGS scores using both methods of assessment (FGS-RT and FGS-IMG). Similarly, in mice and rats, the administration of buprenorphine alone had no impact on the MGS and RGS scores (Miller et al.

Peer) reviewing PDF | (2019:09:40854:3:0:NEW 18 Mar 2020) 
359

360

361

362

363

364

365

366

367

368

369

370

371

372

373

374

375

376

377

378

379

380

381

382

383

384

385

386

387

388

389

390

391

392

393

394

395

396

397

2015; Leung et al. 2016). Even if the real-time evaluation (sedation scores and FGS-RT) might have been biased by the fact that the observer was aware of premedication, this type of bias is not present when using FGS-IMG scores. It is very unlikely that an observer could memorize all cat faces given the number of subjects, timepoints and the long delay between real-time assessment and evaluation of images after blinding and randomization. The action unit "Orbital tightening" was influenced by sedation using the FGS-RT. This effect might be related to the effect of acepromazine causing enophthalmos leading to protrusion of the third eyelid (Hatch et al. 1984) and buprenorphine causing mydriasis (Steagall et al. 2014). This influence should be minimal in the clinical setting, since the final score was not affected by sedation and such changes were not detected using FGS-IMG.

The influence of OVH was determined by comparing the FGS scores 24 hours after extubation with baseline values, in view of the long duration of action of the drugs used herein. Under this condition, OVH did not affect FGS scores (both FGS-RT and FGS-IMG). At this time-point we also would not expect high pain scores considering the minimal degree of surgical manipulation and the use of multimodal analgesia. Mean FGS-RT scores were significantly higher than baseline from 1 to 12 hours after extubation; however, the mean scores remained below the analgesic threshold ( $\geq 0.39$ out of 1.0 ) and the majority of the cats did not require rescue analgesia. Drugs such as acepromazine, buprenorphine, bupivacaine and meloxicam are long-acting drugs, thus their effects would still be present during the postoperative period. In cats, increased sedation scores have been reported for up to 4 hours after intramuscular administration of similar doses of acepromazine and buprenorphine (Hunt et al. 2013).

Furthermore, the mean elimination half-life of bupivacaine was $4.8 \pm 2.7$ and $10.5 \pm 10.3$ hours after intraperitoneal administration of bupivacaine alone and in combination with

dexmedetomidine, respectively, in cats (Benito et al. 2016, 2018). Significant differences in pain or sedation scores, and the prevalence of rescue analgesia were not found between cats receiving intraperitoneal bupivacaine alone or in combination with dexmedetomidine (Benito et al. 2019). For this reason, the FGS scores considered in the present study were analyzed together. Meloxicam was administered at the $12 \mathrm{~h}$ time-point (except if a cat required rescue analgesia before it) and it has a serum half-life of approximately 24 hours (Lehr et al. 2010). Even though the effect of other drugs may have worn off, the results observed at 24 hours after extubation may have been influenced by the long duration of action of meloxicam.

Responsiveness to rescue analgesia, as part of construct validity testing, was previously assessed during the development and validation of the FGS using various analgesic protocols including different drugs, doses and routes of administration (Evangelista et al. 2019). In that study, FGS-IMG scores decreased after the administration of analgesics when compared with those at presentation (i.e. before interventional analgesia). In the present study, responsiveness was tested again since the study design included a standardized protocol for rescue analgesia and type of surgical stimulus $(\mathrm{OVH})$. Additionally, responsiveness should also be assessed using FGS-RT. Both methods of pain assessment (FGS-RT and FGS-IMG) detected changes in pain

Peer) reviewing PDF | (2019:09:40854:3:0:NEW 18 Mar 2020) 
398

399

400

401

402

403

404

405

406

407

408

409

410

411

412

413

414

415

416

417

418

419

420

421

422

423

424

425

426

427

428

429

430

431

432

433

434

435

436

437

scores corroborating our previous findings (Evangelista et al. 2019). Decreases in pain scores were also observed with the SF-UBCPS.

During real-time scoring the observer was present in front of the cage and was able to move around to look at the cat from different angles, while videos were recorded simultaneously from a single angle. The cats' faces were not always visible from the camera angle when screenshots were taken, thus explaining missing FGS-IMG scores in many cases. Indeed, it was not possible to obtain any image from 160 videos (approximately 40\%) for the following reasons: movement of the cat either too close or far from the front of the cage) and out of view; when cats were facing the back of the cage or hiding behind the litter box, sleeping, grooming, or when the image was blurred. Perhaps the number of excluded images could be reduced by using two cameras, placed on either side of the cage, as reported in mice (Langford et al. 2010), rats (Sotocinal et al. 2011) and horses (Dalla Costa et al. 2014). The effect of the presence of the observer in front of the cage while the videos were recorded was not evaluated in this study; however, it is currently being investigated. A recent study in mice showed that the presence of a male observer in the room reduced MGS scores, whereas the same was not observed with female observers. These differences in MGS scores are likely a result of stress-induced analgesia (Sorge et al. 2014). Similarly, the presence of a female observer did not interfere with RGS scores in rats (Leung et al. 2016). In the present study, a female observer performed pain assessments and it remains unknown if the gender of the observer influences FGS scores in cats.

Some limitations of this study include the use of a single type of surgical painful stimulus and model of acute pain $(\mathrm{OVH})$. Effects of sedation were assessed 15 minutes after premedication, before the surgery and any other painful stimulus and only one protocol for premedication was studied. Therefore, our findings are still limited to OVH involving premedication and mild sedation with acepromazine-buprenorphine. It is not clear how other sedative protocols producing moderate to profound sedation may affect the FGS scores. In addition, it was not possible to evaluate the effects of anesthesia and surgery separately on FGS scores since we did not include sham and negative control groups undergoing general anesthesia without the surgical procedure, or surgery without the administration of analgesics (the latter for ethical reasons). This is a clinical study involving surgery that requires the administration of anesthetics; therefore, it is an intrinsic limitation of the methodology. The effects of general anesthesia alone on pain scores were assessed in rats and horses. Higher RGS scores were observed in rats in the immediate time period, approximately 20 minutes after the discontinuation of short duration isoflurane anesthesia (Miller et al. 2016); however, Horse Grimace Scale scores were unchanged 8 hours after general anesthesia without the application of a nociceptive stimulus (Dalla Costa et al. 2014).

\section{Conclusions}

Real-time scoring using the FGS is feasible, although it slightly overestimates image assessment. The minimal bias and narrow limits of agreement between FGS-RT and FGS-IMG 
438 suggest minimal clinical impact. Sedation with acepromazine-buprenorphine and

439 ovariohysterectomy using a multimodal analgesia and a balanced anesthetic protocol did not

440 influence the FGS scores. Responsiveness to analgesic administration was detected both with

441 FGS-RT and FGS-IMG.

442

443 Acknowledgements

444

445

446

447

\section{References}

448

Benito J, Evangelista MC, Doodnaught GM, Watanabe R, Beauchamp G, Monteiro BP, Steagall

449 P. Analgesic Efficacy of Bupivacaine or Bupivacaine-Dexmedetomidine After Intraperitoneal

450 Administration in Cats: A Randomized, Blinded, Clinical Trial. Front Vet Sci. 2019; 6: 307. doi:

451

452 10.3389/fvets.2019.00307

453

Benito J, Monteiro B, Beaudry F, Lavoie AM, Lascelles BD, Steagall PV. Pharmacokinetics of bupivacaine after intraperitoneal administration in cats undergoing ovariohysterectomy. Am J

454 Vet Res. 2016; 77:641-5. doi: 10.2460/ajvr.77.6.641

455

456

Benito J, Monteiro B, Beaudry F, Steagall P. Efficacy and pharmacokinetics of bupivacaine with 457 epinephrine or dexmedetomidine after intraperitoneal administration in cats undergoing ovariohysterectomy. Can J Vet Res. 2018; 82:124-30.

459

460

Bland JM, Altman DG. Agreement between methods of measurement with multiple observations

461

462

463

464 per individual. J Biopharm Stat. 2007; 17:571-582. doi: 10.1080/10543400701329422

\section{5}

Buisman M, Wagner MC, Hasiuk MM, Prebble M, Law L, Pang DS. Effects of ketamine and alfaxalone on application of a feline pain assessment scale. J Feline Med Surg. 2016; 18(8):643-

466

467

468

469

51. doi: 10.1177/1098612X15591590

470

471

Buisman M, Hasiuk MMM, Gunn M, Pang DSJ. The influence of demeanor on scores from two validated feline pain assessment scales during the perioperative period. Vet Anaesth Analg. 2017;44(3):646-655. doi: 10.1016/j.vaa.2016.09.001

472

Dalla Costa E, Minero M, Lebelt D, Stucke D, Canali E, Leach MC. Development of the Horse

473 Grimace Scale (HGS) as a pain assessment tool in horses undergoing routine castration. PLoS

474 One. 2014; 9(3):e92281. doi: 10.1371/journal.pone.0092281

475

Evangelista MC, Watanabe R, Leung VSY, Monteiro BP, O’Toole E, Pang DSJ, Steagall PV.

476 Facial expressions of pain in cats: the development and validation of a Feline Grimace Scale. Sci 477 Rep. 2019; 9:19128. doi:10.1038/s41598-019-55693-8 
478

479

480

481

482

483

484

485

486

487

488

489

490

491

492

493

494

495

496

497

498

499

500

501

502

503

504

505

506

507

508

509

510

511

512

513

514

515

516

Finka LR, Luna SP, Brondani JT, Tzimiropoulos Y, McDonagh J, Farnworth MJ, Ruta M, Mills DS. Geometric morphometrics for the study of facial expressions in non-human animals, using the domestic cat as an exemplar. Sci Rep. 2019; 9(1):9883. doi: 10.1038/s41598-019-46330-5.

Hatch RC, Zahner JM, Booth NH. Meperidine-acepromazine-pentobarbital anesthesia in cats: reversal by 4-aminopyridine and yohimbine. Am J Vet Res. 1984; 45(12):2658-62.

Holden E, Calvo G, Collins M, Bell A, Reid J, Scott EM, Nolan AM. Evaluation of facial expression in acute pain in cats. J Small Anim Pract. 2014;55(12):615-21. doi:

$10.1111 /$ jsap. 12283

Hunt JR, Grint NJ, Taylor PM, Murrell JC. Sedative and analgesic effects of buprenorphine, combined with either acepromazine or dexmedetomidine, for premedication prior to elective surgery in cats and dogs. Vet Anaesth Analg. 2013; 40(3):297-307. doi: 10.1111/vaa.12003

Hunt JR, Knowles TG, Lascelles BD, Murrell JC. Prescription of perioperative analgesics by UK small animal veterinary surgeons in 2013. Vet Rec. 2015; 176:493. doi: 10.1136/vr.102834

Langford DJ, Bailey AL, Chanda ML, Clarke SE, Drummond TE, Echols S, Glick S, Ingrao J, Klassen-Ross T, Lacroix-Fralish ML, Matsumiya L, Sorge RE, Sotocinal SG, Tabaka JM, Wong D, van den Maagdenberg AM, Ferrari MD, Craig KD, Mogil JS. Coding of facial expressions of pain in the laboratory mouse. Nat Methods. 2010; 7(6):447-9. doi: 10.1038/nmeth.1455

Lehr T, Narbe R, Jöns O, Kloft C, Staab A. Population pharmacokinetic modelling and simulation of single and multiple dose administration of meloxicam in cats. J Vet Pharmacol Ther. 2010; 33:277-286. doi: 10.1111/j.1365-2885.2009.01134.x

Leung V, Zhang E, Pang DS. Real-time application of the Rat Grimace Scale as a welfare refinement in laboratory rats. Sci Rep. 2016; 6:31667. doi: 10.1038/srep31667

Lorena SE, Luna SP, Lascelles BD, Corrente JE. Current attitudes regarding the use of perioperative analgesics in dogs and cats by Brazilian veterinarians. Vet Anaesth Analg. 2014; 41(1):82-9. doi: 10.1111/vaa.12104

Mantha S, Roizen MF, Fleisher LA, Thisted R, Foss J. Comparing methods of clinical measurement: reporting standards for bland and altman analysis. Anesth Analg. 2000; 90(3):593602. doi: 10.1097/00000539-200003000-00018 
517 Miller A, Kitson G, Skalkoyannis B, Leach M. The effect of isoflurane anaesthesia and

518 buprenorphine on the mouse grimace scale and behaviour in CBA and DBA/2 mice. Appl Anim

519 Behav Sci. 2015; 172:58-62. doi: 10.1016/j.applanim.2015.08.038

520

521 Miller AL, Golledge HD, Leach MC. The Influence of Isoflurane Anaesthesia on the Rat

522 Grimace Scale. PLoS One. 2016; 11(11):e0166652. doi: 10.1371/journal.pone.0166652

523

524 Miller AL, Leach MC. The Mouse Grimace Scale: A Clinically Useful Tool? PLoS One. 2015;

525 10(9):e0136000. doi: 10.1371/journal.pone.0136000

526

527

528

Morales-Vallecilla C, Ramírez N, Villar D, Díaz MC, Bustamante S, Ferguson D. Survey of Pain Knowledge and Analgesia in Dogs and Cats by Colombian Veterinarians. Vet Sci. 2019; 6(1).

529 doi: $10.3390 /$ vetsci6010006

530

531

Prkachin KM. Assessing pain by facial expression: facial expression as nexus. Pain Res Manag.

532 2009;14(1):53-58. doi:10.1155/2009/542964

533

534

Reimann J, Dewey C, Bateman SW, Kerr C, Johnson R. Perioperative analgesic use by Ontario 535 veterinarians, 2012. Can Vet J. 2017; 58(2):149-156.

536

537 Sorge RE, Martin LJ, Isbester KA, Sotocinal SG, Rosen S, Tuttle AH, Wieskopf JS, Acland EL, 538 Dokova A, Kadoura B, Leger P, Mapplebeck JC, McPhail M, Delaney A, Wigerblad G,

539 Schumann AP, Quinn T, Frasnelli J, Svensson CI, Sternberg WF, Mogil JS. Olfactory exposure

540 to males, including men, causes stress and related analgesia in rodents. Nat Methods. 2004;

541 11:629-632. doi: 10.1038/nmeth.2935

542

543 Sotocinal SG, Sorge RE, Zaloum A, Tuttle AH, Martin LJ, Wieskopf JS, Mapplebeck JC, Wei P,

544 Zhan S, Zhang S, McDougall JJ, King OD, Mogil JS. The Rat Grimace Scale: a partially

545 automated method for quantifying pain in the laboratory rat via facial expressions. Mol Pain.

$546 \quad 2011 ; 7: 55$. doi: 10.1186/1744-8069-7-55

547

548 Steagall PV, Monteiro-Steagall BP, Taylor PM. A review of the studies using buprenorphine in 549 cats. J Vet Intern Med. 2014; 28(3):762-770. doi:10.1111/jvim.12346

550

551 Steagall PV, Taylor PM, Rodrigues LC, Ferreira TH, Minto BW, Aguiar AJ. Analgesia for cats

552 after ovariohysterectomy with either buprenorphine or carprofen alone or in combination. Vet

553 Rec. 2009; 164(12):359-63. doi: 10.1136/vr.164.12.359

554 
555 White WB, Berson AS, Robbins C, Jamieson MJ, Prisant LM, Roccella E, Sheps SG. National 556 standard for measurement of resting and ambulatory blood pressures with automated

557 sphygmomanometers. Hypertension. 1993; 21:504-9. doi: 10.1161/01.hyp.21.4.504 


\section{Figure 1}

\section{Illustration and description of the Feline Grimace Scale}

The Feline Grimace Scale is composed of five action units (ear position, orbital tightening, muzzle tension, whiskers change and head position), each one is scored from 0 to 2 ( $0=$ action unit is absent; $1=$ moderate appearance of the action unit, or uncertainty over its presence; and 2 = obvious appearance of the action unit).

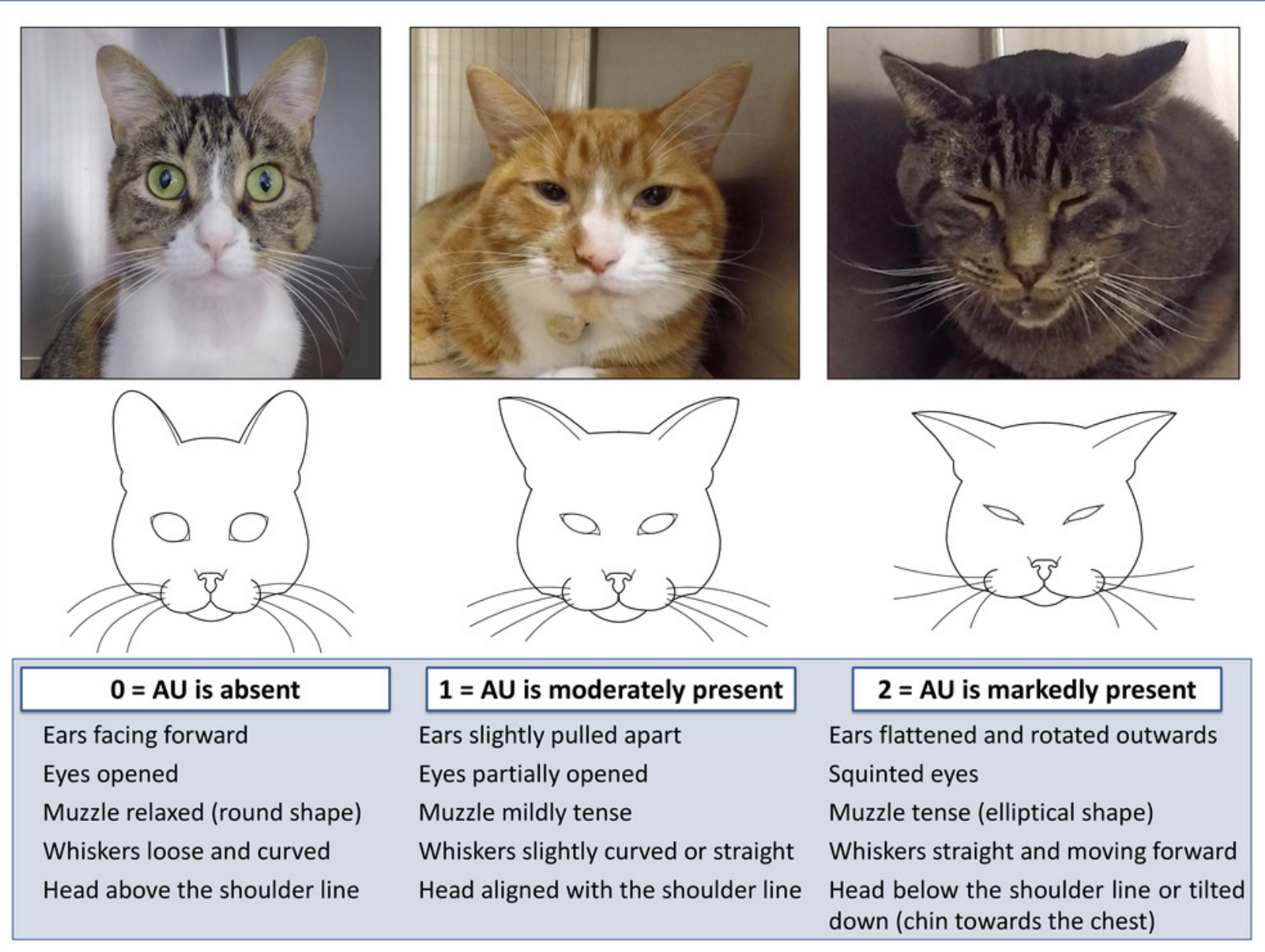


Figure 2

Timeline of the study and the time-points for sedation and pain assessments in realtime and video-recordings

Sedation was evaluated with a 5-point simple descriptive scale (SDS). Pain was evaluated using the Feline Grimace Scale (FGS) and the short-form UNESP-Botucatu composite pain scale (SF-UBCPS). FGS scores were obtained in real time (FGS-RT) during three minutes of observation of the cats undisturbed. Three-minute videos were recorded simultaneously to the FGS-RT assessment using a GoPro camera placed between the cage bars.

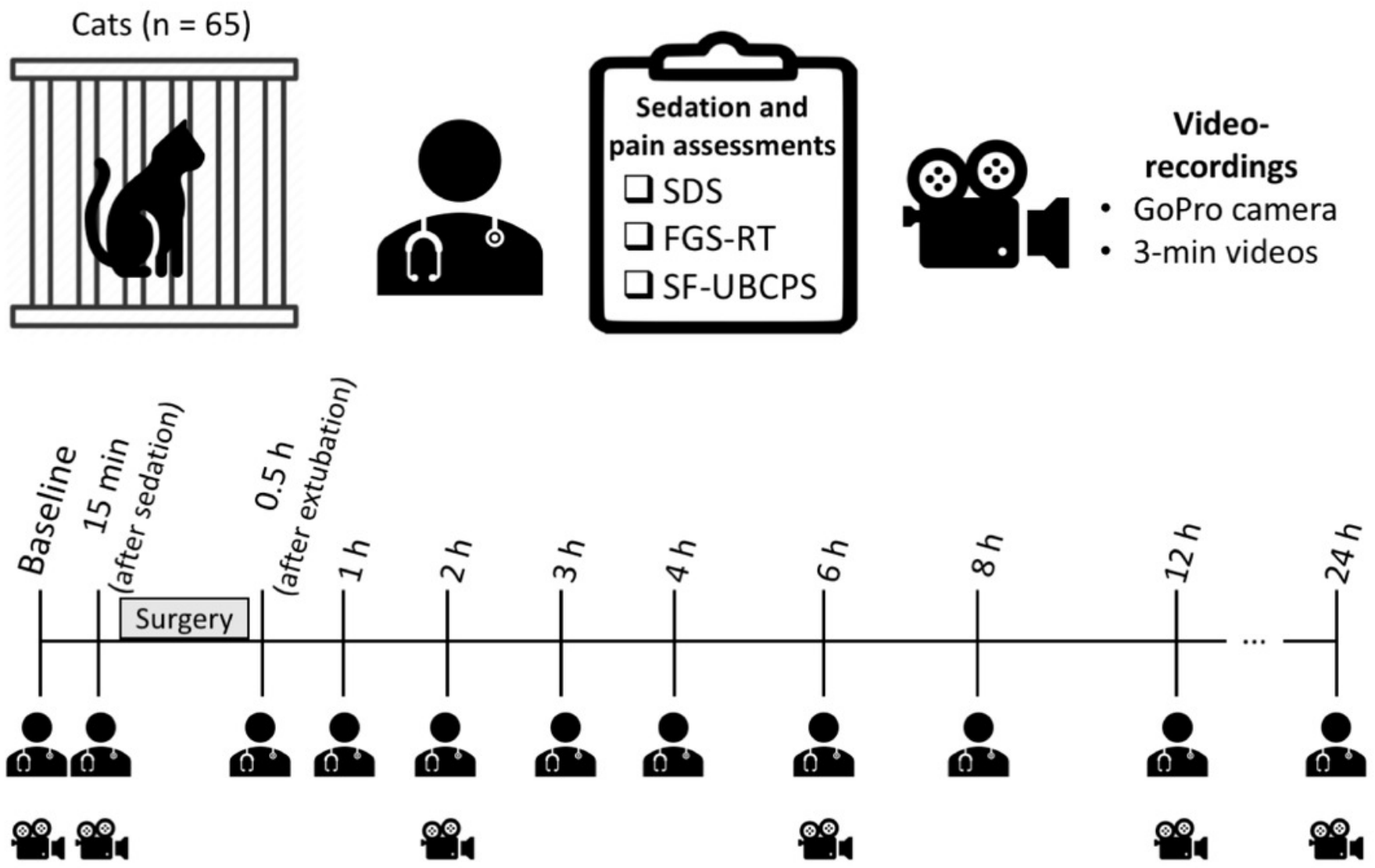




\section{Figure 3}

Agreement between Feline Grimace Scale scores obtained in real-time (FGS-RT) and by image assessment (FGS-IMG)

Bland \& Altman's plots showing the bias and limits of agreement (LOA) between FGS-RT and FGS-IMG for the A) First minute of observation; B) Second minute of observation; C) Third minute of observation and D) Final score (average of the three minutes). Numbers of pairs of scores used for each analysis are indicated below the charts. 


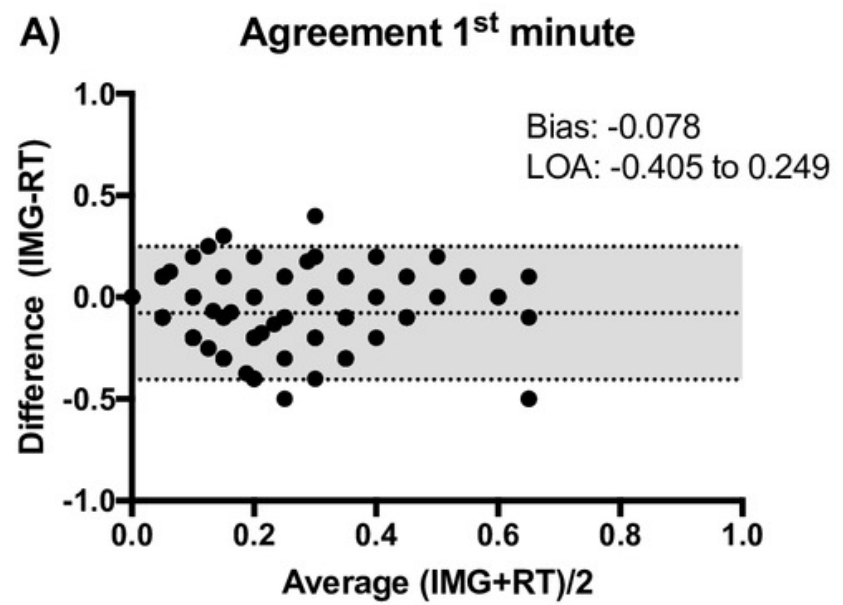

$n=170$ pairs of observations

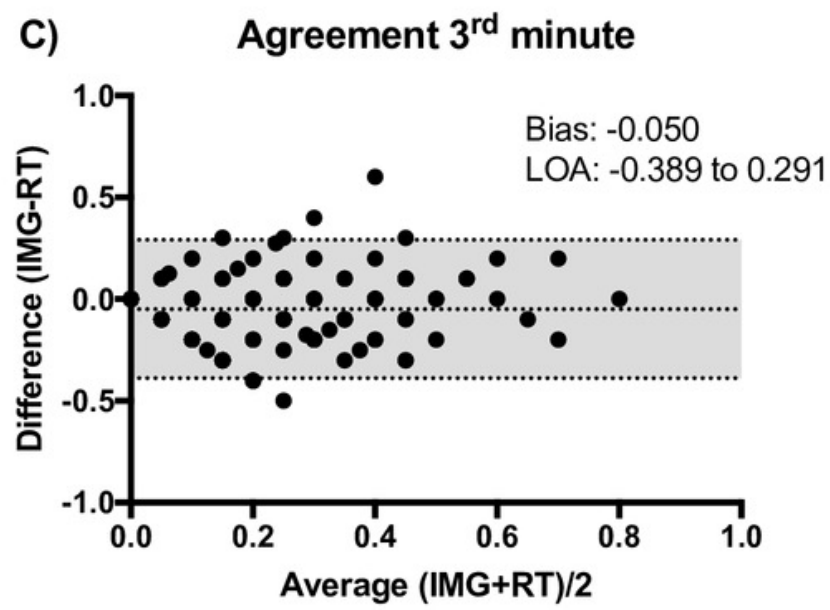

$n=158$ pairs of observations
B) Agreement $2^{\text {nd }}$ minute

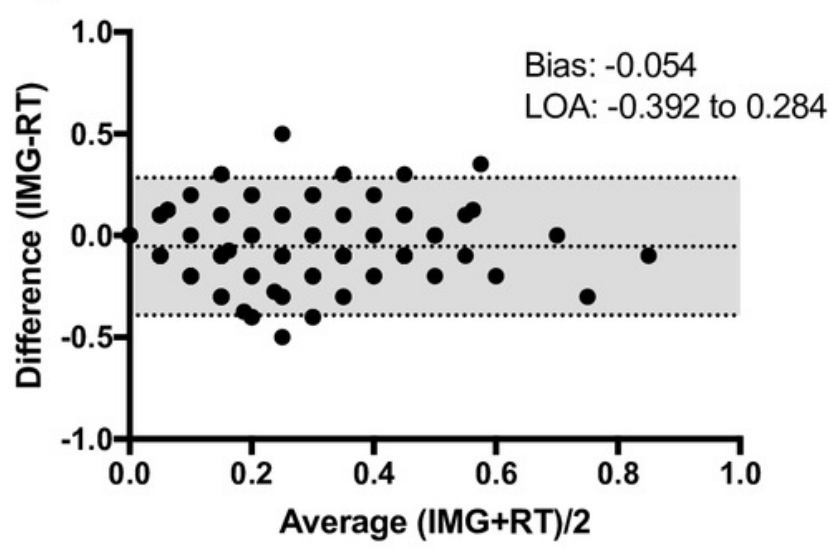

$n=167$ pairs of observations

D) Agreement Final score

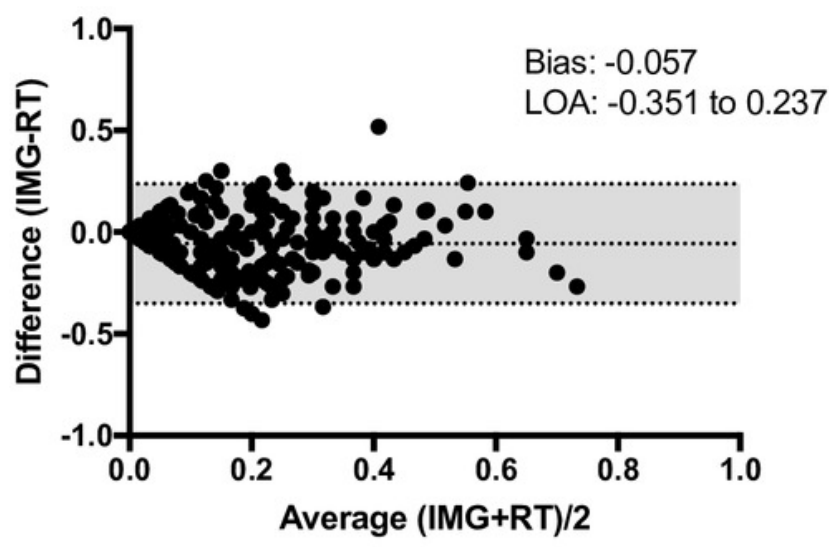

$n=232$ pairs of observations 


\section{Figure 4}

Mean \pm SEM Feline Grimace Scale scores in real-time (FGS-RT) in cats undergoing ovariohysterectomy

Scores were obtained pre- and postoperatively (Pre-op and Post-op, respectively) at baseline, 15 minutes after sedation with acepromazine-buprenorphine, and between 0.5 to 24 hours after extubation. The average score of three minutes of observation of the cats' facial expression was considered the final FGS score per time-point. *Significantly different from baseline scores $(p<0.05)$.

\section{Pain}

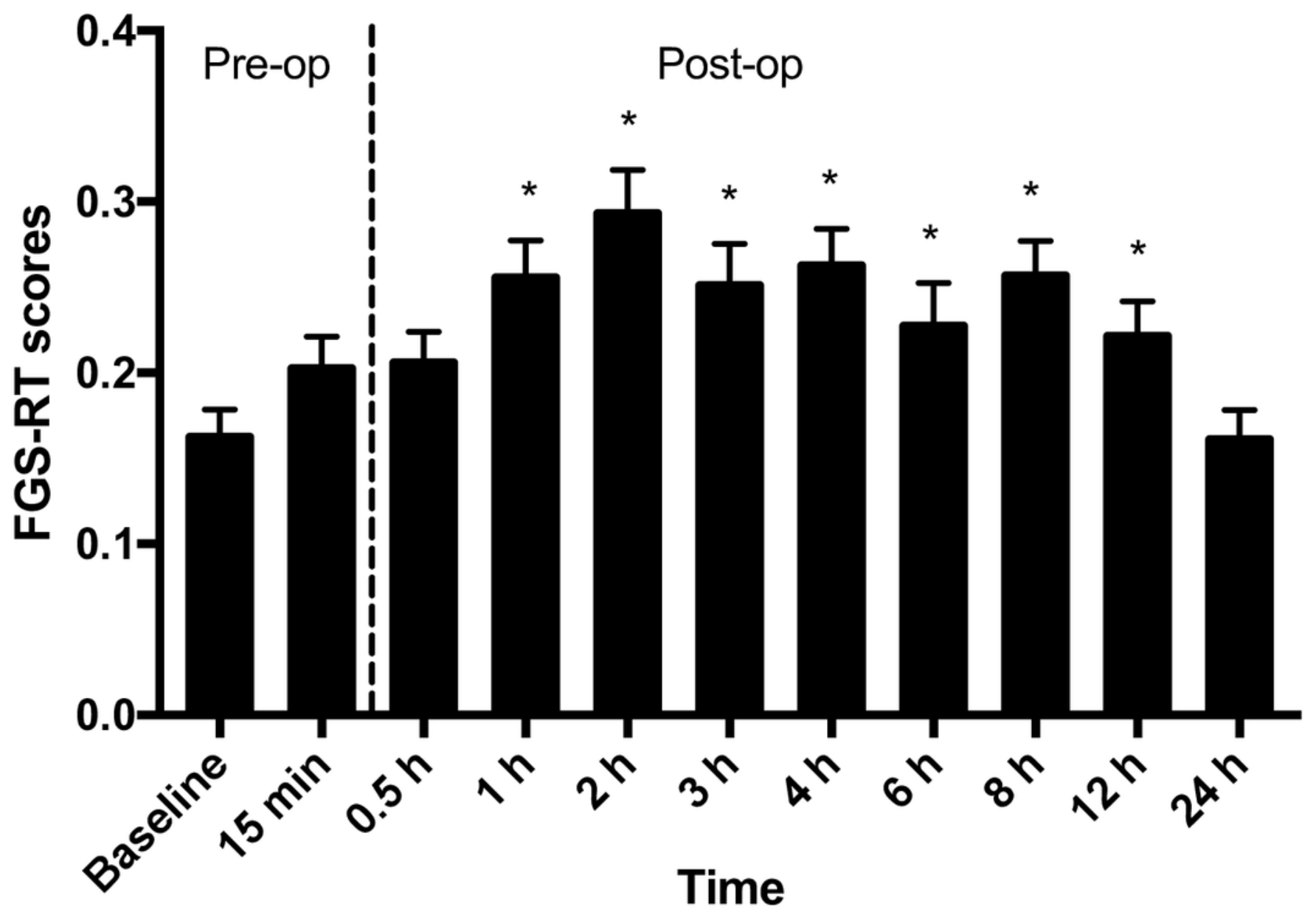




\section{Table $\mathbf{1}$ (on next page)}

Sedation scores obtained over time using a 5-point simple descriptive scale (adapted from Steagall et al. 2009).

Scores were obtained at baseline, 15 minutes after sedation with acepromazinebuprenorphine, and between 0.5 to 24 hours after extubation. Scores over time were compared with baseline using Friedman test and Dunn's multiple comparisons test. Sedation scores are presented as median (range) and interquartile range. Median of the differences (in comparison with baseline) and their $95 \%$ confidence interval $(\mathrm{Cl})$ are presented.

*Significantly different from baseline $(p<0.05)$. 
1 Table 1. Sedation scores obtained over time using a 5-point simple descriptive scale 2 (adapted from Steagall et al. 2009).

3

\begin{tabular}{cccccc}
\hline & \multicolumn{2}{c}{ Sedation scores } & & & \\
\cline { 2 - 4 } Time-point & Median (range) & $\begin{array}{c}\text { Interquartile } \\
\text { range }\end{array}$ & $\begin{array}{c}\text { Median of } \\
\text { differences }\end{array}$ & $\begin{array}{c}\text { 95\% CI of the } \\
\text { differences }\end{array}$ & $p$ value \\
\hline Baseline & $0(0-2)$ & {$[0-0]$} & - & - & - \\
15 min & $1(0-2)^{*}$ & {$[0-2]$} & 0 & 0 to 1 & 0.012 \\
$0.5 \mathrm{~h}$ & $1(0-3)^{*}$ & {$[1-1]$} & 1 & 1 to 1 & $<0.001$ \\
$1 \mathrm{~h}$ & $1(0-3)$ & {$[0-2]$} & 0 & 0 to 1 & 0.053 \\
$2 \mathrm{~h}$ & $1(0-3)$ & {$[0-2]$} & 0 & 0 to 1 & 0.051 \\
$3 \mathrm{~h}$ & $0(0-3)$ & {$[0-2]$} & 0 & 0 to 0 & 0.161 \\
$4 \mathrm{~h}$ & $0(0-3)$ & {$[0-2]$} & 0 & 0 to 0 & 0.155 \\
$6 \mathrm{~h}$ & $0(0-3)$ & {$[0-2]$} & 0 & 0 to 0 & $>0.999$ \\
$8 \mathrm{~h}$ & $0(0-3)$ & {$[0-2]$} & 0 & 0 to 0 & $>0.999$ \\
$12 \mathrm{~h}$ & $0(0-3)$ & {$[0-0]$} & 0 & 0 to 0 & $>0.999$ \\
$24 \mathrm{~h}$ & $0(0-2)$ & {$[0-0]$} & 0 & 0 to 0 & $>0.999$ \\
\hline
\end{tabular}

4

5 Scores were obtained at baseline, 15 minutes after sedation with acepromazine-buprenorphine, 6 and between 0.5 to 24 hours after extubation. Scores over time were compared with baseline 7 using Friedman test and Dunn's multiple comparisons test. Sedation scores are presented as 8 median (range) and interquartile range. Median of the differences (in comparison with baseline) 9 and their $95 \%$ confidence interval (CI) are presented. *Significantly different from baseline $(p<$ 10 0.05). 


\section{Table 2 (on next page)}

Mean \pm SD Feline Grimace Scale scores in real-time (FGS-RT) in cats undergoing ovariohysterectomy.

Scores were obtained at baseline, 15 minutes after sedation with acepromazinebuprenorphine, and between 0.5 to 24 hours after extubation. Scores over time were compared with baseline using linear mixed models. The mean differences (in comparison with baseline) and their $95 \%$ confidence interval $(\mathrm{Cl})$ are presented. *Significantly different from baseline $(p<0.05)$. 
1 Table 2: Mean \pm SD Feline Grimace Scale scores in real-time (FGS-RT) in cats undergoing 2 ovariohysterectomy.

3

\begin{tabular}{ccccc}
\hline & FGS-RT scores & & & \\
\cline { 2 - 3 } Time-point & Mean \pm SD & Mean difference & $\begin{array}{c}95 \% \text { CI of the } \\
\text { differences }\end{array}$ & $p$ value \\
\hline Baseline & $0.16 \pm 0.13$ & - & - & - \\
15 min & $0.2 \pm 0.15$ & 0.04 & 0.0045 to 0.0755 & 0.3847 \\
$0.5 \mathrm{~h}$ & $0.21 \pm 0.14$ & 0.047 & 0.0028 to 0.0920 & 0.2624 \\
$1 \mathrm{~h}$ & $0.26 \pm 0.17^{*}$ & 0.097 & 0.0462 to 0.1480 & 0.0003 \\
$2 \mathrm{~h}$ & $0.29 \pm 0.2^{*}$ & 0.132 & 0.0824 to 0.1820 & $<0.0001$ \\
$3 \mathrm{~h}$ & $0.25 \pm 0.18^{*}$ & 0.092 & 0.0434 to 0.1410 & 0.0003 \\
$4 \mathrm{~h}$ & $0.26 \pm 0.16^{*}$ & 0.106 & 0.0605 to 0.1520 & $<0.0001$ \\
$6 \mathrm{~h}$ & $0.23 \pm 0.18^{*}$ & 0.075 & 0.0277 to 0.1230 & 0.0095 \\
$8 \mathrm{~h}$ & $0.26 \pm 0.14^{*}$ & 0.097 & 0.0454 to 0.1480 & 0.0001 \\
$12 \mathrm{~h}$ & $0.22 \pm 0.14^{*}$ & 0.059 & 0.0096 to 0.1090 & 0.0316 \\
$24 \mathrm{~h}$ & $0.16 \pm 0.12$ & $<0.0001$ & -0.0434 to 0.0434 & 0.9998 \\
\hline
\end{tabular}

4

5 Scores were obtained at baseline, 15 minutes after sedation with acepromazine-buprenorphine, 6 and between 0.5 to 24 hours after extubation. Scores over time were compared with baseline 7 using linear mixed models. The mean differences (in comparison with baseline) and their 95\% 8 confidence interval (CI) are presented. *Significantly different from baseline $(p<0.05)$. 9 